\title{
Late Presenters of HIV Infection in an HIV Unit of a Tertiary University Hospital in a Rural Region of Greece
}

\author{
Vasileios Petrakis, Periklis Panagopoulos, Tzelal Maltsan, Anna-Maria Xanthopoulou, Irene Terzi, \\ Nikolaos Papanas, Efstratios Maltezos, and Dimitrios Papazoglou
}

\begin{abstract}
A significant progress has been made over the years in the prognosis and treatment of patients with early diagnosis of HIV infection. However, late presentation of a large number of patients remains a serious public health problem. The aim of our study is to highlight the dimensions of the problem by evaluating the data from the HIV Unit of Alexandroupolis, a rural region with population heterogeneity and a strategic position between West and East, Europe, and Asia. This was a retrospective study, including 107 patients diagnosed with HIV infection in our unit from 2010 to 2018 . Late presenters (LP) were defined as patients diagnosed with a CD4 cell count $<350 / \mathrm{mm}^{3}$ or an AIDS-defining condition regardless of CD4 cell count. The proportion of patients diagnosed late was $49.5 \%$. The majority were males in the age group 31-40 years (41.5\%). Men who had sex with men were 37.8\%. Among LP, 34\% were at Center for Disease Prevention and Control stage C3. The most common AIDS-defining condition observed was Pneumocystis jirovecii Pneumonia (15.1\%), followed by esophageal candidiasis (7.5\%) and cryptococcal meningitis (3.8\%). In addition, immune reconstitution inflammatory syndrome was documented (3.8\%). A high percentage of patients were also coinfected with hepatitis B (22.6\%) virus. The notably high percentage of LP in our unit demonstrates that late presentation remains a challenge for public health. Further efforts must be made to ensure an early diagnosis of HIV infection. The early initiation of antiretroviral therapy is vital to reduce viral load to undetectable levels and the risk of HIV transmission.
\end{abstract}

Keywords: late presenters, HIV, AIDS, HIV epidemic, PLHIV, AIDS-defining conditions

\section{Introduction}

A CCORDING TO THE SUSTAINABLE development goals, the aim is termination of HIV epidemic by 2030 (Ref. $^{1}$ ). However, the reality is less optimistic, given that $49 \%$ of people living with HIV (PLHIV) diagnosed in 2017 in the European region were late presenters (LP), including $28 \%$ with advanced HIV infection. ${ }^{2}$ LP are defined as patients presenting for care with a CD4 cell count below 350 cells $/ \mathrm{mm}^{3}$ or with an AIDS-defining event regardless of the CD4 cell count. There is also the term "presentation with advanced HIV disease" for patients presenting for care with a CD4 cell count below 200 cells $/ \mathrm{mm}^{3}$ or with an AIDS-defining event regardless of the CD4 cell count. ${ }^{3}$

There are many negative consequences of late presentation not only for patients themselves but also for public health. Patients with early diagnosis and initiation of antiretroviral therapy (ART) can achieve a life expectancy similar to the general population. ${ }^{4}$ By contrast, LP have a higher mortality rate, even during the first year of diagno- sis. ${ }^{5}$ They are diagnosed too late for a significantly effective treatment. They are more likely to die due to AIDS-defining conditions despite the initiation of ART immediately after HIV diagnosis. ${ }^{6}$ A low current CD4 cell count is also associated with a higher incidence of non-AIDS-defining malignancies. ${ }^{7}$ In addition, late presentation leads to a particularly increased risk of HIV transmission, because LP for very long are unaware of their HIV infection. ${ }^{8}$ The risk of HIV transmission from unaware HIV infected people is at least thrice higher than people diagnosed early. ${ }^{9}$ People who know their HIV status tend to reduce risk behaviors associated with an increased possibility of transmission. ${ }^{9}$

Late presentation and diagnosis of HIV infection remains an ultimate challenge for Greece, as well. Among those diagnosed with HIV infection in 2017 with the available CD4 cell count at diagnosis, $56.7 \%$ were LP, including $35.9 \%$ with advanced HIV disease. ${ }^{10}$

Our HIV unit covers a regional area (East Macedonia and Thrace) with multiple specific characteristics. It is a rural area, with population heterogeneity (minorities, refugees, 
immigrants), while it is located in a cardinal point, between West and East, Europe and Asia. Stigma is still a major consideration, as well as access in check points (rapid test spots). Thus, the aim of the present study was to evaluate the problem of LP in our clinic and highlight the importance of early diagnosis and linkage to a care center.

\section{Patients and Methods}

This was a retrospective cohort study conducted in the HIV Unit of the University General Hospital of Alexandroupolis (Greece). During a period of 8 years 107 individuals newly diagnosed with HIV infection were included in the study. Data on gender, age, transmission mode, CD4 cell count at the moment of diagnosis, date of first positive HIV test, presence of AIDS-defining diseases, and clinical condition at diagnosis were collected. The study was carried out in accordance with the Helsinki Declaration of Human Rights, and patients gave their informed consent.

Patients were classified as non-LP or LP. The number of LP with advanced HIV infection was estimated as well. Persons with CD $4 \leq 350$ cells $/ \mathrm{mm}^{3}$ or diagnosed with AIDS regardless of CD4 cell count were defined as LP, while persons with CD $4 \leq 200$ cells $/ \mathrm{mm}^{3}$ or AIDS were defined as LP with advanced HIV disease. ${ }^{3}$ Patients were also classified with 1993 Center for Disease Prevention and Control (CDC) classification system for HIV infection. ${ }^{11}$

For each patient, transmission mode was determined at diagnosis. Patients were divided into four categories: men who have sex with men (MSM), heterosexual contact, injection drug users, and unspecified risk group. It also examined the proportion of patients with coexisting hepatitis $\mathrm{B}$ virus (HBV) or hepatitis $\mathrm{C}$ virus (HCV) infection or syphilis.

\section{Results}

During the period 2010-2018, 107 patients were diagnosed with HIV infection. The CD4 cell count at the moment of diagnosis was available for all of them. Fifty-three patients were LP (49.5\%), including 36 patients with advanced HIV infection (33.6\%). An increasing trend was observed over the years not only in the number of new diagnoses but also in the number of LP. The number of patients diagnosed in our clinic increased from 7 patients in 2010 to 19 patients in 2017. As for LP, the proportion was $42.9 \%$ in 2010 and $47.4 \%$ in 2017 (Table 1).

Table 1. Trend of New HiV Diagnoses And Late
Presenters During the Period 2010-2018

\begin{tabular}{ccc}
\hline Year & $\begin{array}{c}\text { Total number of new } \\
\text { HIV diagnoses }(\mathrm{n})\end{array}$ & $\begin{array}{c}\text { Proportion } \\
\text { of LP }(\%)\end{array}$ \\
\hline 2010 & 7 & 42.9 \\
2011 & 8 & 50 \\
2012 & 8 & 37.5 \\
2013 & 9 & 40.2 \\
2014 & 11 & 45.5 \\
2015 & 12 & 41.7 \\
2016 & 14 & 42.8 \\
2017 & 19 & 47.4 \\
2018 & 19 & 47.4 \\
\hline
\end{tabular}

$\mathrm{LP}$, late presenters.
There are significant differences between LP and non-LP diagnosed in our unit (Table 2). The median age of diagnosis in this group of patients was 36.5 years old. By contrast, LP were older when diagnosed. MSM were the major risk group with a more significant proportion compared with LP. The proportion of PLHIV that belonged to unspecified risk group or heterosexuals was lower. More than half of the patients were Greek $(83.3 \%)$, single $(68.5 \%)$, orthodox $(88.9 \%)$, and well educated (high school or university, 62.3\%), similar to LP. Three patients of non-LP were refugees, two men from Middle East and a pregnant woman from Africa. The proportion of asymptomatic patients was $85.2 \%$, while more than half of LP had moderate or severe symptoms at the moment of diagnosis.

The majority of LP were males $(81.1 \%)$. The highest percentage of patients was in the age group of 31-40 years $(41.5 \%)$, followed by 12 patients $(22.6 \%)$ diagnosed older than 50 years old and 11 patients $41-50$ years old.

Table 2. Demographic Characteristics of Late Presenters and Nonlate Presenters

\begin{tabular}{|c|c|c|}
\hline & $L P, \mathrm{n}(\%)$ & $\begin{array}{c}\text { Non-LP, } \\
\mathrm{n}(\%)\end{array}$ \\
\hline$N$ & $\begin{array}{c}53 \text { (49.5) (LP with } \\
\text { advanced HIV } \\
\text { infection 36) }\end{array}$ & $54(51.5)$ \\
\hline \multicolumn{3}{|c|}{ (1) } \\
\hline Male & $43(81.1)$ & $46(85.2)$ \\
\hline \multicolumn{3}{|l|}{ Age } \\
\hline Median age, years old & 45 & 36.5 \\
\hline Median range, years old & $21-66$ & $18-73$ \\
\hline 1st Quartile, years old & 39 & 34 \\
\hline 3rd Quartile, years old & 52.5 & 48.5 \\
\hline$<30$ years old & $8(15.1)$ & $10(18.5)$ \\
\hline 30-40 years old & $22(41.5)$ & $21(38.9)$ \\
\hline $41-50$ years old & $11(20.8)$ & $17(31.5)$ \\
\hline$>50$ years old & $12(22.6)$ & $6(11.1)$ \\
\hline \multicolumn{3}{|l|}{ Transmission mode } \\
\hline MSM & $20(37.8)$ & $30(55.6)$ \\
\hline Unspecified & $16(30.2)$ & $10(18.5)$ \\
\hline Heterosexual & $12(22.6)$ & $8(14.8)$ \\
\hline PWID, HCV coinfected & $5(9.4)$ & $6(11.1)$ \\
\hline \multicolumn{3}{|l|}{ Origin } \\
\hline Greece & $47(88.7)$ & $45(83.3)$ \\
\hline Post-Soviet states & $5(9.4)$ & $5(9.3)$ \\
\hline Other countries & $1(1.9)$ & $1(1.8)$ \\
\hline Refugees & $0(0)$ & $3(5.6)$ \\
\hline \multicolumn{3}{|l|}{ Religion } \\
\hline Orthodox & $43(81.1)$ & $48(88.9)$ \\
\hline Muslim & $10(18.9)$ & $6(11.1)$ \\
\hline \multicolumn{3}{|l|}{ Education } \\
\hline Elementary & $20(37.7)$ & $19(35.2)$ \\
\hline High school & $25(47.2)$ & $20(37)$ \\
\hline University & $8(15.1)$ & $15(27.8)$ \\
\hline \multicolumn{3}{|l|}{ Living place } \\
\hline Urban & 39 (73.6) & $17(31.5)$ \\
\hline Rural & $14(26.4)$ & $37(68.5)$ \\
\hline \multicolumn{3}{|l|}{ Status } \\
\hline Married & $13(24.5)$ & $46(85.2)$ \\
\hline Single & $40(75.6)$ & 8 (14.8) \\
\hline
\end{tabular}

HCV, hepatitis C virus; MSM, men who have sex with men; PWID, people who inject drugs. 
The proportion of MSM diagnosed late was $37.8 \%$, significantly lower compared with non-LP. A significant proportion of patients belonged to the unspecified risk group (30.2\%). In 12 patients $(22.6 \%)$, HIV infection was probably heterosexually transmitted. Five LP (9.6\%) diagnosed in our clinic were people who inject drugs (PWID) and HCV coinfected. Demographic characteristics of LP are shown in Table 2.

CDC stage A2 was seen in $26.4 \%$ and A3 in $13.2 \%$ of LP, mainly with asymptomatic HIV infection. Only one patient at stage A presented with persistent generalized lymphadenopathy and two with severe weight loss. Fourteen patients (24.4\%) were at stage $\mathrm{B}$, with moderate symptoms and clinical manifestations already appearing at the moment of diagnosis. The commonest symptoms included oral candidiasis (7.5\%), idiopathic thrombocytopenic purpura (5.7\%), oral hairy leukoplakia (3.8\%), and herpes zoster infection (3.7\%). An extremely high proportion of LP were at stage C3. Eighteen patients (34\%) were LP with advanced HIV infection, as they had CD4 cell count below 200 cells $/ \mathrm{mm}^{3}$ and an AIDS-defining condition (Table 3 ).

Eight $(15.1 \%)$ patients at C3 stage were diagnosed with Pneumocystis jirovecii Pneumonia (PJP) and four (7.5\%) with esophageal candidiasis. Among patients with esophageal candidiasis, one revealed atypical mycobacterial infection. One patient presented with wasting syndrome, one with primary brain lymphoma, and one with cytomegalovirus (CMV) retinitis. In 2017 two individuals newly diagnosed with HIV infection had cryptococcal meningitis at the moment of diagnosis. In addition, newly diagnosed LP after initiation of ART performed immune reconstitution inflammatory syndrome (IRIS) of progressive multifocal leukoencephalopathy and cryptosporidium infection. The majority of LP (60.4\%) were hospitalized when diagnosed due to their clinical condition, with a delay discharge.

More than half of LP $(60.4 \%)$ had no coexisting infection at diagnosis. However, the proportion of patients with $\mathrm{HBV}$

Table 3. Clinical Condition

\begin{tabular}{lcc}
\hline & $\begin{array}{c}\text { LP, } \\
\mathrm{n}(\%)\end{array}$ & $\begin{array}{c}\text { Non-LP, } \\
\mathrm{n}(\%)\end{array}$ \\
\hline Stage A & $21(39.6)$ & $46(85.2)$ \\
Asymptomatic HIV infection & $18(34)$ & $46(85.2)$ \\
Weight loss & $2(3.8)$ & \\
Persistent generalized & $1(1.9)$ & \\
lymphadenopathy & & \\
Stage B & $14(26.4)$ & $8(14.8)$ \\
Oral candidiasis & $4(7.5)$ & $4(7.4)$ \\
Idiopathic thrombocytopenic & $3(5.7)$ & $2(3.7)$ \\
purpura & $3(5.7)$ & $2(3.7)$ \\
Oral hairy leukoplakia & $2(3.8)$ & \\
Diarrhea, fever, fatigue $>1$ month & $2(3.8)$ & \\
Varicella-Zoster virus infection & $18(34)$ & $0(0)$ \\
Stage C & $8(15.1)$ & \\
PJP & $4(7.5)$ & \\
Esophageal candidiasis & $2(3.8)$ & \\
Cryptococcal meningitis & $1(1.9)$ & \\
CMV retinitis & $1(1.9)$ & \\
Wasting syndrome & $1(1.9)$ & \\
Primary brain lymphoma & $1(1.9)$ & \\
PML & 53 & 54 \\
Total & &
\end{tabular}

CMV, cytomegalovirus; PJP, Pneumocystis jirovecii Pneumonia; PML, progressive multifocal leukoencephalopathy. infection was high $(22.6 \%)$. Five PLHIV were diagnosed with HCV infection (7.5\%), while $3.8 \%$ of LP had $\mathrm{HBV} / \mathrm{HCV}$ coinfection (all intravenous drug users). There were also three patients diagnosed with syphilis (5.7\%).

Despite the late presentation and delayed linkage to care, the majority of LP maintained at least $95 \%$ adherence to the ART. Only four patients didn't have a good compliance and two stopped their therapy.

\section{Discussion}

Despite the significant progress in prognosis and treatment of patients diagnosed early with HIV infection, late presentation remains a serious public health problem. Almost half of patients $(49.5 \%)$ diagnosed with HIV infection in our clinic were LP. Significantly high is also the percentage of newly diagnosed patients with advanced HIV infection (33.6\%). Taking into consideration the prevalence of LP in the European countries in 2017 (49\%), the results of our study demonstrate the dimensions of the problem. ${ }^{2}$ We are far from The Joint United Nations Programme on HIV/AIDS (UNAIDS) target 90\%$90 \%-90 \%$, as the estimated number of people living unaware of their HIV status in the European Union/European Economic Area (EU/EEA) remains high with the median time from infection to diagnosis to be $\sim 3$ years. ${ }^{12,13}$ There is a low but notable reduction in prevalence of LP probably due to an improvement in testing strategies and linkage to care. ${ }^{14}$

Our results are lower compared with the corresponding proportions in Greece, where $56.7 \%$ of PLHIV diagnosed in 2017 were LP, including $35.9 \%$ with advanced HIV infection. ${ }^{10}$ The proportion of LP in our study is lower as our unit covers a rural area, and thus, there is the opportunity to check soon after the diagnosis people who could be infected by newly diagnosed patients. There is also a low number of PWID limiting this possible transmission mode. The commonest AIDS-defining disease observed in Greece was PJP, like in our unit, followed by wasting syndrome. ${ }^{10}$ In our study, PJP was followed by esophageal candidiasis.

The problem of LP is huge throughout Europe. According to the French Hospital Database on HIV during the period 2003-2009, 53.9\% of patients newly diagnosed with HIV infection were LP. As in other studies, it was observed that MSM were less likely to present late compared with other risk groups. ${ }^{15}$ In our study similar findings were documented. The percentage of LP is extremely high in Georgia, as well. Fortunately, during the period 2012-2015 a declining trend has been documented especially among MSM. ${ }^{16}$ Hence, the number of new diagnoses per year is increased. ${ }^{16}$ The most common risk group diagnosed late in this study was injection drug users in contrast to our clinic in which PWID is the less common risk group diagnosed late. ${ }^{16}$ One of the highest percentages of LP in Europe was observed in Poland. During the period 2006-2017, 62.86\% of newly diagnosed patients were LP demonstrating the need of improving and expanding testing strategies not only for MSM group. ${ }^{17}$

In a cohort study conducted in the United Kingdom, it was observed that almost half of the patients (49\%) were LP, and the study highlights the association of late presentation with heterosexual contact, older age, and non-Caucasian origin. ${ }^{18}$ The same risk groups were associated with a higher possibility of late presentation and in a retrospective cohort study in Belgium. The percentage of LP was $44 \%$ with a significant 
number of refugees among them. ${ }^{19}$ Alexandroupolis is a city next to the Greek-Turkish borders, a crossroad of three continents: Asia, Africa, and Europe, and so the intermediate station for many refugees or immigrants to pass to their final destination, other European countries. Three refugees have been diagnosed in our unit who were not LP, two men from Middle East and a pregnant woman from Africa. After the confirmation of diagnosis they were transferred to Athens. The highest proportion of migrants among LP in European countries was observed in Sweden, mainly infected before arrival. ${ }^{20}$

The first step is to understand the risk factors associated with late presentation. Studies have reported that LP tend to belong to groups not characterized as high risk for HIV infection. In such patients, transmission mode is likely to remain unknown, ${ }^{21}$ similar to our series. We have also observed an increasing trend of patients tested several times for HIV infection aware of their possible risk behavior. These patients, mainly MSM, were diagnosed early and are classified among non-LP $(50.5 \%)$. Other reasons of late presentation are the unwillingness of patients for diagnosis due to the fear of HIVrelated stigma and the delayed testing as individuals, not well informed of HIV infection, don't feel ill. ${ }^{22} \mathrm{~A}$ high proportion of LP are late testers although they belong among known high risk groups. In PWID the corresponding proportion is lower due to their association with various health programs, but the linkage with a care center is late. ${ }^{23}$ The problem with injection drug users is similar in Greece. During 2011-2013 an extreme increase of HIV infections occurred in Athens (the Capital) among PWID. ${ }^{10}$ One potential reason is the no distribution of sterile syringes to injection drug users. The Needle and Syringe Programs have been proven effective in reducing HIV transmission and coinfections, but the access to programs providing medical care and social support is also vital. ${ }^{24-26}$ In our unit, the percentage of PWID was $9.4 \%$ among LP with no significant increase during the HIV outbreak observed in Greece in 2011-2013.

Although the number of MSM diagnosed late with HIV infection in our clinic was higher than other risk groups, a notable decline was observed, while the proportion of LP among people with heterosexual contact or injection was increasing. In 2014 five patients diagnosed late were MSM. In 2017 four out of nine LP were heterosexuals and one was injecting drugs. In 2018 five out of nine LP were heterosexuals. In the National Observation HIV cohort study ATHENA conducted in the Netherlands had observed a similar decline for MSM diagnosed as LP. ${ }^{27}$ Conversely, for heterosexual males and females the percentages of LP remained high. ${ }^{27}$ Similar results had been also documented in a prospective observational study in Edinburg. The proportion of heterosexual males diagnosed late was $45 \%$, while the proportion of MSM was lower. ${ }^{28}$ The observation that the risk of late presentation is increased among heterosexuals while a decline was observed among MSM has to be highlighted. That is probably due to better information and screening of MSM population. In contrast, heterosexuals are more likely to have unsafe sex although some of them are well informed.

Another important issue is the large number of LP with HBV or HCV or even with both HBV/HCV coinfections. This high prevalence of $\mathrm{HCV}$ coinfection among injection drug users has been proven in other studies as well. ${ }^{26}$ These coinfections have a negative impact on the clinical progress of HIV infection. HBV coinfected individuals have an increased risk, almost double, of AIDS-defining diseases and mortality compared with patients without $\mathrm{HBV}$ coinfection. ${ }^{29}$ In addition, HIV infection leads to higher levels of HBV DNA and an increasing risk of cirrhosis and hepatocellular carcinoma. ${ }^{30}$ Choice of ART and clinical monitoring of patients with coinfections should be very careful to avoid drug interactions and possible toxicity, a usual challenge and problem in this group of patients. $^{31,32}$ It is important to choose regimen taking into consideration the clinical condition of patient and possible drug interactions and the probable high pill burden. ${ }^{32}$ LP are more likely to develop drug toxicities and IRIS or hepatitis flare. ${ }^{33}$

ART has achieved an important progress in prognosis of patients early diagnosed with HIV infection. Mortality from AIDS-defining diseases has been reduced, while there is an increase in the non-AIDS related deaths, especially malignancies. ${ }^{34}$ There is also a significant increase in deaths due to liver diseases more often in HCV coinfected patients who inject drugs. ${ }^{31}$ However, late presentation and presentation with advanced HIV infection remain major prognostic factors leading to high mortality rate mainly due to AIDS-defining diseases. ${ }^{6,34,35}$ According to the results of Collaboration of Observational HIV Epidemiological Research Europe study, $30 \%$ of HIV infected patients were LP with an opportunistic infection at diagnosis. ${ }^{14}$ Thus, the majority of LP was hospitalized at the moment of diagnosis $(60.4 \%)$. In addition to poor prognosis, late presentation is associated with an extremely high cost for medical services even from the first year after diagnosis, due to prolonged hospitalization. ${ }^{36}$

The limitations of our study include the small number of cases and the retrospective character.

\section{Conclusion}

In summary, although there is a declining trend, the percentage of LP and presenters with advanced HIV infection remains high. We are still far from UNAIDS 90\% target. LP represent a serious public health problem and an ultimate challenge. They miss the opportunity to initiate ART early and achieve a life expectancy similar to general population. It is crucial to diagnose them early and to ensure their linkage to care centers immediately after HIV positive testing.

\section{Author Disclosure Statement}

No competing financial interests exist.

\section{Funding Information}

No funding was received for this article.

\section{References}

1. The Joint United Nations Programme on HIV/AIDS (UNAIDS): On the Fast-Track to End AIDS by 2030: Focus on Location and Population. UNAIDS, Geneva, 2015.

2. European Center for Disease Prevention and Control (ECDC)/WHO Regional Office for Europe: HIV/AIDS Surveillance in Europe, 2017 Data. ECDC, Stockholm, 2018.

3. Antinori A, Coenen T, Costagiola D, et al:: Late presentation of HIV infection: A consensus definition. HIV Med 2011;12:61-64.

4. Gueler A, Moser A, Calmy A, et al.: Life expectancy in HIV positive persons in Switzerland: Matched comparison with general population. AIDS 2017;31:427-436. 
5. Brown AE, Kall MM, Smith RD, Yin Z, Hunter A, Delpech VC: Auditing National HIV Guidelines and Policies: The United Kingdom CD4 Surveillance Scheme. Open AIDS J 2012;6:149-155.

6. Sobrino-Vegas P, Moreno S, Rubio R, et al.: Impact on late presentation of HIV infection on short-, mid- and longterm mortality and causes of death in a multicenter national cohort: 2004-2013. J Infect 2016;72:587-596.

7. Reekie J, Kosa C, Engsig F, et al.: Relationship between current level of immunodeficiency and non-acquired immunodeficiency syndrome-defining malignancies. Cancer 2017;116:5306-5315.

8. Hall HI, Holtgrave DR, Maulsby C: HIV transmission rates from persons living with HIV who are aware and unaware of their infection. AIDS 2012;26:893-896.

9. Marks G, Crepaz N, Jansen RS: Estimating sexual transmission of HIV from persons aware and unaware that they are infected with the virus in the USA. AIDS 2006;20:1447-1450.

10. Annual Edition of Hellenic Center for Disease Control and Prevention (HCDCP): Epidemiological Data of HIV Infection, 2017 Data. HCDCP, Greece, 2018.

11. Center for Disease Control and Prevention: 1993 revised classification system for HIV infection and expanded surveillance case definition for AIDS among adolescents and adults. MMWR Recomm Rep 1992;41(RR-17):1-19.

12. The Joint United Nations Programme on HIV/AIDS (UNAIDS): 90-90-90: An Ambitious Treatment Target to Help End the AIDS Epidemic. UNAIDS, Geneva, 2014.

13. van Sighem A, Pharris A, Quinten C, Noori T, AmatoGauci AJ: ECDC HIV/AIDS surveillance and Dublin declaration monitoring networks. Eurosurveillance 2017;22: 17-00771.

14. Chêne G, Phillips A, Costagliola D: Cohort Profile: Collaboration of Observational HIV Epidemiological Research Europe (COHERE) in EuroCoord. Int J Epidemiol 2016;46: 797-797n.

15. Montlahuc C, Guiguet M, Abgrall S, et al.: Impact of late presentation on the risk of death among HIV- infected people in France (2003-2009). J AIDS 2013;64:197-203.

16. Chkhartshvili $\mathrm{N}$, Chokoshvili $\mathrm{O}$, Bolokadze $\mathrm{N}$, et al.: Late presentation of HIV infection in the country Georgia: 2012-2015. PLoS One 2017;12:e0186835.

17. Wojcik-Cichy K, Jablonowska O, Piekarska A, Janlonowska E: The high incidence of late presenters for HIV/AIDS infection in the Lodz province, Poland in the years 2009-2016: We are still far from the UNAIDS 90\% target. AIDS Care 2018;30:1538-1541.

18. Iwuji CC, Churchill D, Gilleece Y, Weiss H, Fisher M: Older HIV-infected individuals present late and have a higher mortality: Brighton, UK cohort study. BMC Public Health 2013;13:397.

19. Darcis G, Lambert I, Sauvage A-S, et al.: Factors associated with late presentation for HIV care in a single Belgian reference center: 2006-2017. Sci Rep 2018;8:8594.

20. Brannstrom J, Svedhem Johansson V, Marrone G, et al.: Deficiencies in the health care system contribute to a high rate of HIV diagnosis in Sweden. HIV Medicine 2016;17:425-435.

21. Delpierre C, Lauwers-CancesV, Pugliese P, et al.: Characteristics trends, mortality and morbidity in persons newly diagnosed HIV positive during the last decade: The profile of new HIV diagnosed people. Eur J Public Health 2008; 18:345-347.

22. Bonjour MA, Montagne M, Zambrano M, et al.: Determinants of late disease-stage presentation at diagnosis of HIV infection in Venezuela: A case-case comparison. AIDS Res Ther 2008;5:6.

23. Couturier E, Schwoebel V, Michon C, et al.: Determinants of delayed diagnosis of HIV infection in France, 19931995. AIDS 1998;12:795-800.

24. Raffetti E, Concetta Postorino M, Castelli F, et al.: The risk of late or advanced presentation of HIV infected patients is still high, associated factors evolve but impact on overall mortality is vanishing over calendar years: Results from the Italian MASTER Cohort. BMC Public Health 2016;16:878.

25. Fernades RM, Cary M, Duarte G, et al.: Effectiveness of needle and syringe Programmes in people who inject drugs-An overview of systematic reviews. BMC Public Health 2017;17:309.

26. Page K, Morris MD, Hahn JA, Maher L, Prins M: Injection drug USE and hepatitis $\mathrm{C}$ virus infection in young adult injectors: using evidence to inform comprehensive prevention. Clin Infect Dis 2013;57:32-38.

27. Op de Coul EL, van Sighem A, Brinkman K, et al.: Factors associated with presenting late or with advanced HIV infection in the Netherlands, 1996-2014: Results from a national observational study. BMJ Open 2016;6:e009688.

28. Mavani K, McMillan A, Ogilvie M, Scott G: Heterosexual men and women with HIV test positive at a later stage of infection than homo- or bisexual men. Int J STD AIDS 2004; $15: 811-814$.

29. Chun HM, Roediger MP, Huppler Hullsiek K, et al.: Hepatitis B virus coinfection negatively impacts HIV outcomes in HIV seroconverters. J Infect Dis 2012;205:185-193.

30. Thio $\mathrm{Cl}$ : Hepatitis $\mathrm{B}$ and human immunodeficiency coinfection. Hepatology 2009;49:138-145.

31. Rockstroh JK, Soriano V, Tural C, et al.: Influence of hepatitis $\mathrm{C}$ virus infection on HIV-1 disease progresseion and response to highly active antiretroviral therapy. J Infect Dis 2005; 192:992-1002.

32. Sulkowski MS, Thomas DL, Chasisson RE, Moore RD: Hepatotoxicity associated with antiretroviral therapy in adults infected with human immunodeficiency virus and the role of hepatitis C or B virus infection. JAMA 2000;283:74-80.

33. Shelburne SA, Visnegarwala F, Darcourt J, et al.: Incidence and risk factors for immune reconstitution inflammatory syndrome during highly active antiretroviral therapy. AIDS 2005;19:399-406.

34. Mocroft A, Brettle R, Kirk O, et al:: Changes in the cause of death among HIV positive subjects across Europe: Results from the EuroSIDA study. AIDS 2002;16:1663-1671.

35. Weber R, Ruppik M, Rickenbach M, et al.: Decreasing mortality and changing patterns of causes of death in the Swiss HIV Cohort Study. HIV Med 2013;14:195-207.

36. Krentz HB, Auld MC, Gill MJ: The high cost of medical care for patients who present late $(\mathrm{CD} 4<200$ cells $/ \mathrm{mL})$ with HIV infection. HIV Med 2004;5:93-98.

Address correspondence to: Vasileios Petrakis HIV Unit

2nd Department of Internal Medicine University General Hospital of Alexandroupolis Democritus University of Thrace Alexandroupolis 68100 Greece

E-mail: vasilispetrakis1994@gmail.com 